
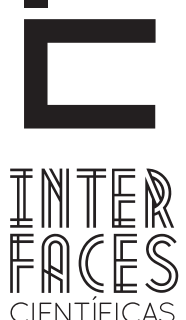

SAÚDE E AMBIENTE

\title{
RETINOBLASTOMA: EPIDEMIOLOGIA E SOBREVIDA EM SERGIPE
}

Eldon Bezerra da Silva Junior ${ }^{1}$

\section{RESUMO}

O Objetivo é descrever a epidemiologia e a sobrevida por retinoblastoma dos pacientes oncológicos pediátricos cadastrados nos serviços públicos de oncologia pediátrica em Aracaju, Sergipe. 0 método - Foram analisados os prontuários de 1203 pacientes pediátricos oncológicos cadastrados nos dois principais serviços de oncologia pediátrica de Aracaju, no período de 1980 a 2010. Destes, foram selecionados aqueles pacientes com idade de até 20 anos, com diagnóstico de retinoblastoma. Os resultados foram apresentados em números absolutos e proporções (intervalo de confiança de 95\%). A sobrevida foi avaliada através do método de Kaplan-Meier. Resultados: Dos 54 pacientes que entraram neste estudo $49(90,7 \%)$ eram provenientes do estado de Sergipe. Do total de casos, a maior parte $(64,8 \%)$ foi proveniente do interior do estado. A prevalência foi maior no gênero feminino
(53,7\%). A faixa etária mais frequente foi a compreendida entre zero a quatro anos correspondendo a 49 casos (90,7\%). 0 sintoma mais freqüente foi a mancha em olho $(75,9 \%)$. A maior parte dos pacientes $(53,7 \%)$ teve o diagnóstico após três meses do inicio dos sintomas. Observou-se que 27 pacientes (50\%) evoluíram para o óbito. A sobrevida em cinco anos foi de 47,2\%. Conclusões: As características clínicas e epidemiológicas do retinoblastoma encontrados neste estudo estão de acordo com o encontrado na literatura mundial. A sobrevida por retinoblastoma no estado de Sergipe é inferior à encontrada no Brasil e em países desenvolvidos.

\section{PALAVRAS CHAVE}

Retinoblastoma. Epidemiologia. Sobrevida. 


\section{ABSTRACT}

The Objective is to describe the epidemiology and survival of retinoblastoma in pediatric oncology patients registered at pediatric oncology public hospitals of Aracaju, Sergipe. Method: The medical records of 1203 patients, registered at two main pediatric oncology services of Aracaju, in the period from 1980 to 2010 , were analyzed. Of these, we selected those patients aged up to 20 years, diagnosed with retinoblastoma. The results were presented in absolute numbers and proportions (confidence interval of 95\%). Survival was evaluated by Kaplan-Meier method. Results: Of the 54 patients who entered this study, 49 (90.7\%) were from the state of Sergipe. Of all cases, the majority $(64.8 \%)$ came from the countryside. The prevalence was higher in females ( $53.7 \%)$. The most common age group was between zero and four years accounting for 49 cases $(90.7 \%)$. The most frequent symptom was spot on eye (75.9\%). Most patients (53.7\%) was diagnosed three months after onset of symptoms. It was observed that 27 patients (50\%) subsequently died. The five-year survival was $47.2 \%$. Conclusions: The clinical and epidemiological characteristics of retinoblastoma in this study are consistent with findings in the world literature. Survival for retinoblastoma in the state of Sergipe is lower than that found in Brazil and in developed countries.

\section{KEYWORDS}

Retinoblastoma. Epidemiology. Survival.

\section{RESUMEN}

El objetivo es describir la epidemiología y la supervivencia por retinoblastoma en los pacientes oncológicos pediátricos matriculados en los servivios de oncología pediátrica en Aracaju, Sergipe. Métodos: Se revisaron las historias clínicas de 1203 pacientes pediátricos oncológicos incluidos en los dos principales servicios de oncología pediátrica de Aracaju, en el período de 1980 a 2010. De éstos, se seleccionaron los pacientes de hasta 20 años, con diagnóstico de retinoblastoma. Los resultados se presentaron en números absolutos y proporciones (intervalo de confianza del 95\%). La supervivencia se evaluó mediante Kaplan-Meier. Resultados: De los 54 pacientes que entraron en el estudio $49(90,7 \%)$ eran del estado de Sergipe. De todos los casos, la mayoría $(64,8 \%)$ procedían del campo. La prevalencia fue mayor en mujeres $(53,7 \%)$. El grupo de edad más frecuente fue entre cero y cuatro años totalizando 49 casos $(90,7 \%)$. El síntoma más frecuente fue la mancha en el ojo (75,9\%). La mayoría de los pacientes $(53,7 \%)$ se diagnosticó tres meses después de la aparición de los síntomas. Se observó que 27 pacientes (50\%) murieron posteriormente. La supervivência en cinco años fue de 47,2\%. Conclusiones: Las características clínicas y epidemiológicas del retinoblastoma en este estudio son consistentes con los resultados en la literatura. La supervivencia de retinoblastoma en el estado de Sergipe es menor que la encontrada en Brasil y en los países desarrollados.

\section{PALABRAS CLAVES}

Descriptores. Retinoblastoma. Epidemiología. Supervivencia.

\section{INTRODUÇ̄̃O}

0 retinoblastoma é o tumor intraocular mais comum na infância e corresponde a $11 \%$ de todos os cânceres que ocorrem durante o primeiro ano de vida
(CHOI et al, 2010); acomete 1/15000 a 1/20000 nascidos vivos por ano (AERTS et al. 2006). Apesar de $90 \%$ das crianças afetadas viverem em países subdesen- 
volvidos, a literatura destas regiões sobre o retinoblastoma é escassa (CANTURK et al, 2010).

Desde os primeiros relatos do tratamento de crianças com retinoblastoma, a sobrevivência destes pacientes tem aumentado dramaticamente. Este câncer mortal no século XX foi o câncer pediátrico com a maior taxa de sobrevivência em 2003 (NASERIPPOUR et al, 2009). O sucesso do tratamento do retinoblastoma depende da habilidade dos pais e do pediatra em detectar a doença quando ela ainda é intraocular, encaminhando-a precocemente ao oftalmologista para a realização de um fundo de olho e

\section{MÉTODOS}

0 presente estudo é longitudinal, observacional e descritivo. Foi feita a coleta e análise retrospectiva de dados dos prontuários de 1203 pacientes pediátricos oncológicos cadastrados nos serviços de oncologia pediátrica do Centro de Oncologia Dr. Osvaldo Leite (Hospital Governador João Alves Filho) e Fundação Beneficente Hospital de Cirurgia no período de 1980 a 2010. Destes, foram selecionados aqueles pacientes com idade de até 20 anos, com diagnóstico de retinoblastoma, comprovado pelos meios disponíveis nesses serviços. Foi construído um banco de dados através do programa SPSS versão 17.0. Os dados co-

\section{RESULTADOS}

Foram incluídos neste estudo 54 prontuários e destes, $49(90,7 \%)$ eram provenientes do estado de Sergipe e cinco $(9,3 \%)$ de outros estados. Os pacientes provenientes de municípios do interior do estado de Sergipe compreenderam 35 casos, ou seja, 64,8\% da amostra. A maior parte, $29(53,7 \%)$ era constituída do gênero feminino. A faixa etária mais frequente foi a compreendida entre zero ao oncologista pediátrico para tratamento adequado ao estádio da doença.

A despeito dos esforços de médicos e voluntários para aumentar a detecção precoce do retinoblastoma, os estudos tem mostrado pouca evolução em relação à redução do tempo entre o início dos sintomas e a procura pelo médico e também em relação à rapidez no estabelecimento do diagnóstico e do tratamento adequado (RODRIGUES et al, 2004). O presente estudo objetiva descrever a epidemiologia e a sobrevida por retinoblastoma dos pacientes oncológicos pediátricos cadastrados nos serviços públicos de Oncologia Pediátrica em Aracaju, Sergipe.

letados foram analisados através do mesmo programa estatístico. Os resultados foram apresentados em números absolutos e proporções (intervalo de confiança de $95 \%$ ). A sobrevida foi avaliada através do método de Kaplan-Meier, sendo consideradas significativas as diferenças cujas chances de exclusão da hipótese $\mathrm{HO}$ sejam superiores a $95 \%(p<0,05)$.

Este projeto foi submetido e aprovado em Comitê de Ética em Pesquisa envolvendo Seres Humanos da Universidade Federal de Sergipe, sob o número CAAE 0024.0.107.000-09 em 13 de abril de 2009.

a quatro anos correspondendo a 49 casos $(90,7 \%$ ) (Tabela 1). Quanto à topografia do tumor, o olho esquerdo foi mais acometido com 21 casos (38,9\%), e 15 (27,8\%) tiveram acometimento de ambos os olhos (Tabela 2).

Os sinais e sintomas mais frequentes foram a mancha em olho em 41 pacientes (75,9\%), a tumoração 
em olho em oito (14,8\%), estrabismo em dois $(3,7 \%)$, secreção em olho em dois (3,7\%) e dor em um (1,9\%) paciente. Vinte e nove pacientes $(53,7 \%)$ tiveram diagnostico com mais de três meses do aparecimento dos sintomas. 0 tratamento mais frequente foi a associação de cirurgia com quimioterapia em 34 casos (63\%), ficando em segundo lugar o tratamento com cirurgia, quimioterapia e radioterapia associados em 17 pacientes (31,5\%). Observou-se que 27 pacientes (50\%) evoluíram para o óbito (Tabela 3).
A sobrevida global nos primeiros 60 meses foi de $47,2 \%$ (Figura 1). Não foi encontrada diferença com significância estatística na sobrevida em 60 meses quando comparamos os pacientes da capital (Aracaju) e do interior $(p=0,718)$. Também não houve diferença na sobrevida em 60 meses entre os gêneros ( $p=0,279)$.

Tabela 1 - Faixas etárias dos pacientes portadores de retinoblastoma

\begin{tabular}{cccc}
\hline Variável & $\mathbf{n}$ & \% & IC \\
\hline 0-4anos & 49 & 90,7 & $83,3-98,1$ \\
5-9anos & 3 & 5,6 & $0-13$ \\
$10-14$ anos & 1 & 1,9 & $0-5,6$ \\
>15anos & 1 & 1,9 & $0-5,6$ \\
\hline
\end{tabular}

Tabela 2 - Topografia do tumor em pacientes portadores de retinoblastoma.

\begin{tabular}{cccc}
\hline Variável & $\mathbf{n}$ & $\mathbf{\%}$ & IC \\
\hline Olho esquerdo & 21 & 38,9 & $24,1-51,9$ \\
Olho direito & 18 & 33,3 & $20,4-46,3$ \\
Ambos os olhos & 15 & 27,8 & $16,7-38,9$ \\
\hline
\end{tabular}

Tabela 3 - Evolução dos pacientes portadores de retinoblastoma

\begin{tabular}{cccc}
\hline Variável & $\mathbf{n}$ & $\mathbf{\%}$ & IC \\
\hline Óbito & 27 & 50 & $37-63$ \\
Cura & 11 & 20,4 & $11,1-31,5$ \\
Abandono* $^{\text {Em controle }}$ & 7 & 13 & $3,7-22,2$ \\
\hline
\end{tabular}

*Pacientes que abandonaram o tratamento. 


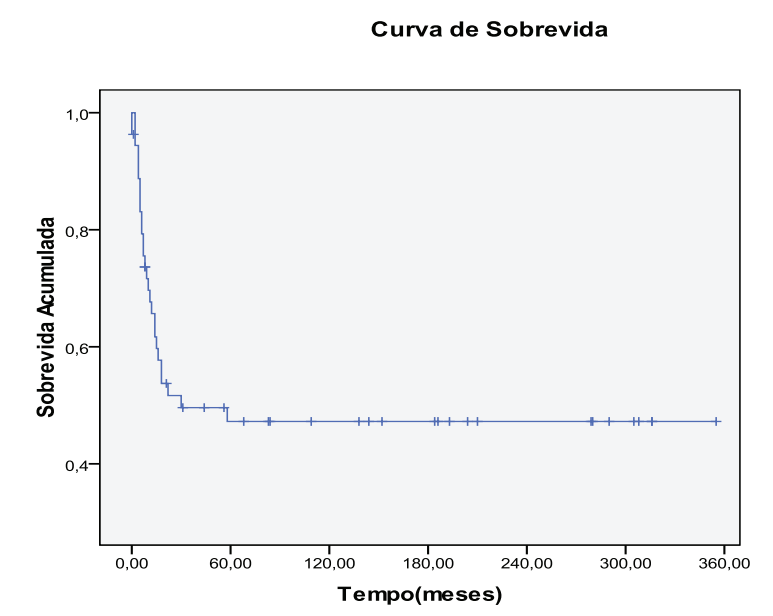

$\neg$ Curva de Sobrevida + Censurados

\section{DISCUSSÃO}

Embora o retinoblastoma seja um tumor relativamente incomum, ocorre numa proporção de 1:15000 a 1:20000 nascidos vivos (SHIELDS et al, 2004). Um estudo que utilizou 14 registros de câncer de base populacional do Brasil mostrou que o retinoblastoma teve uma incidência relativamente alta em quase todos os registros. Esse estudo constatou que entre crianças de zero a quatro anos de idade, as maiores taxas de incidência ajustadas pela idade foram encontradas em Natal (27/milhão), Jaú (23/milhão), Goiânia (21,5/ milhão), São Paulo (21,5/milhão), Porto Alegre (19,4/ milhão), Manaus (15/milhão) e Salvador (14,6/mi(hão). Em Aracaju a taxa de incidência ajustada pela idade em crianças com 19 anos ou menos foi de 5,43/ milhão (CAMARGO et al, 2010).

Rodrigues et al (2004) estudou 327 pacientes atendidos no Hospital do Câncer de São Paulo e encontrou que a maioria $(52,3 \%)$ eram do sexo masculino. Bonanomi et al (2009), analisou 28 novos casos de retinoblastoma do Hospital das Clínicas da Faculdade de Medicina da Universidade de São Paulo e não houve diferença na incidência do retinoblastoma entre os gêneros.

Segundo Szilárd et al (2008) a incidência de retinoblastoma hereditário é constante entre as várias populações do mundo e não há predileção por gênero. Febrônio et al (2000) estudou 8 pacientes cadastrados no Serviço de Oncologia do Hospital Governador João Alves Filho, em Sergipe, no período de 1994-1999, e encontrou uma frequência maior do retinoblastoma no sexo masculino. No presente estudo o gênero mais frequente foi o feminino, correspondendo a $53,7 \%$, porém 
esta diferença não teve significância estatística, concordando assim com o encontrado na literatura.

A leucocoria e o estrabismo são as manifestações clínicas mais frequentes do retinoblastoma (AERTS et al, 2006). Nesse estudo o sintoma mais frequente também foi a leucocoria, seguido pela tumoração em olho, estrabismo, secreção em olho, e dor ocular.

Esse estudo mostrou que a faixa etária mais frequente foi a compreendida entre zero e quatro anos. Esse achado está de acordo com o encontrado no estudo de Melo et al (2008) onde a maioria dos casos de retinoblastoma acontece em crianças bastante jovens com $63 \%$ abaixo de dois anos de idade e $95 \%$ antes dos cinco anos. Após esta faixa etária o aparecimento do retinoblastoma é raro.

Em $60 \%$ dos casos o retinoblastoma é unilateral e a forma hereditária corresponde a $15 \%$ da incidência mundial da doença (AERTS et al, 2006). No presente estudo o acometimento unilateral também foi mais frequente, sendo mais comum o acometimento do olho esquerdo. 0 acometimento bilateral também esteve presente em uma porção expressiva da amos$\operatorname{tra}(27,8 \%)$.

O diagnóstico precoce do retinoblastoma continua sendo um desafio tanto em países em desenvolvimento como em países desenvolvidos. Rodrigues et al, (2004) estudou 327 pacientes e constatou que o atraso no diagnóstico (tempo de queixa maior que 6 meses) foi fator prognóstico de pior sobrevida. Um estudo anterior realizado em 139 casos de retinoblastoma encontrou menores taxas de sobrevida em pacientes que tiveram diagnóstico com idade superior a 2 anos (NASERIPOUR et al, 2009). Na amostra desse estudo, a maior parte dos pacientes teve o diagnóstico com mais de 3 meses após o aparecimento dos sintomas.

O tratamento da criança com retinoblastoma é individualizado e baseado na possibilidade de doença metastática, riscos de cânceres secundários, situação sistêmica, lateralidade da doença, tamanho e localização dos tumores, prognóstico visual antecipado, e resposta ao tratamento (SZILÁRD et al, 2008). A doença intraocular continua a ser abordada por um largo número de modalidades de tratamentos incluindo: enucleação, termoterapia transpupilar, crioterapia, fotocoagulação a laser, braquiterapia, radioterapia e quimioterapia (sistêmica ou local) (AERTS et al, 2006). No presente estudo as modalidades de tratamento foram divididas em cirurgia, quimioterapia e radioretapia, estando as mesmas isoladas ou associadas. A modalidade de tratamento mais frequente foi a cirurgia associada à quimioterapia.

A sobrevida em 5 anos dos pacientes com diagnóstico de retinoblastoma nesse trabalho foi de $47,2 \%$. Este valor é inferior ao encontrado hoje em países em desenvolvimento como o Brasil, bem como é inferior ao encontrado em países desenvolvidos. Um estudo publicado em 2011 mostra que o Brasil tem uma sobrevida por retinoblastoma de $78 \%$, ficando atrás de países como Argentina (93\%), Chile (90\%), Uruguai (92\%) e Colômbia (92\%), sendo superior apenas a Equador (66\%) e Peru (60\%) (CANTURK et al, 2010).

No estudo de Febrônio et al (2000) constatou-se que $50 \%$ dos casos de retinoblastoma evoluíram para o óbito. Este dado está de acordo com o encontrado neste estudo, que mostra a mesma taxa de $50 \%$ de óbitos entre os casos de retinoblastoma. Este mau resultado pode, em parte, ser explicado pelo atraso no diagnóstico, que pode ser o responsável pelo pior prognóstico destes pacientes.

Levando em consideração que a maioria dos pacientes, deste estudo, foi diagnosticada após três meses do início dos sintomas, isto sugere que a provável causa da baixa sobrevida seja o atraso diagnóstico.

Neste estudo o tratamento mais utilizado foi a cirurgia associada à quimioterapia. Este dado sugere que os pacientes já chegam aos serviços em estágio avançado da doença e contribui para a suspeita do 
provável atraso diagnóstico. Outra possível causa da alta taxa de óbitos nos primeiros 60 meses seria a agressividade do tratamento, visto que o tratamento mais usado foi a cirurgia com quimioterapia. Porém, este tratamento faz parte do protocolo utilizado na maioria dos serviços no Brasil, nos quais a sobrevida é maior que a encontrada no estado de Sergipe. Logo, este argumento não pode ser utilizado para justificar a baixa sobrevida nesse estudo.
Ao comparar a sobrevida segundo os gêneros, esse estudo não encontrou diferença com significância estatística. Este dado está de acordo com o encontrado na literatura. Comparando os grupos da capital e interior, não foi encontrada diferença com significância estatística na sobrevida. Isto pode ser explicado pelo fato de o estado de Sergipe ter pequenas dimensões e, portanto o acesso aos serviços especializados em Aracaju é relativamente fácil.

\section{CONCLUSÕES}

As características clínicas e epidemiológicas do retinoblastoma encontrados neste estudo estão de acordo com o encontrado na literatura mundial. A sobrevida por retinoblastoma no estado de Sergipe é inferior à encontrada no Brasil e em países desenvolvidos.

\section{REFERÊNCIAS}

AERTS, I. et al. Retinoblastoma. Orphanet Journal of Rare Diseases, v. 1, n. 31, 2006.

ANTONELI, C. B. G. et al. O papel do pediatra no diagnóstico precoce do retinoblastoma. Revista da Associação Médica Brasileira, São Paulo, v. 50, n. 4, p. 400-02, dez. 2004.

BONANOMI, M. T. B. C et al. Retinoblastoma: a three-year-study at a Brazilian medical school hospital. Clinics, V. 64, n, 5, p. 427-34, 2009.

CAMARGO, B. et al. Cancer incidence among children and adolescents in Brazil: first report of 14 population-based cancer registries. International Journal of Cancer, v. 126, n. 3, p. 715-20, 2010.

CANTURK, S. et al. Survival of retinoblastoma in less-developed countries impact of socioeconomic and health-related indicators. British Journal of Ophthalmology, v. 94, n 11, p. 1432-36, nov. 2010.

$\mathrm{CHOI}, \mathrm{S}$. Y. et al. Long term follow-up results of external beam radiotherapy as primary treatment for retinoblastoma. Journal of Korean Medical Science, v.25, n 4, p. 546-51, apr. 2010.

CHUNG, S. E. et al. Clinical manifestations and treatment of retinoblastoma in Korea. British Journal of Ophthalmology, v. 92, n 9, p. 1180-84, 2008. 
FEBRÔNIO, M. V. Retinoblastoma: Revisão dos Últimos Cinco Anos em Sergipe(1994-1999). Aracaju, 2000.

KLEINERMAN, R. A. et al. Risk of new cancers after radiotherapy in long-term survivors of retinoblastoma: An extended follow-up. Journal of Clinical Oncology, v. 23, n 10, p. 2272-79, 2005.

MELO, M. C. S. C. et al. Retinoblastoma bilateral de aparecimento tardio: relato de caso. Arquivos Brasileiros de Oftalmologia, v. 71, n 3, 437-42, mai-jun. 2008.

NASERIPOUR, M. et al. Retinoblastoma Survival in Iran: 10 Years Experience of a Referal Center. Iranian Journal of Ophthalmology, v. 21, n. 4; p.17-24, 2009.

ORJUELA, M. et al. Presence of human papilloma virus in tumor tissue from children with retinoblastoma: an alternative mechanism for tumor development, Clinical Cancer Research, v. 6, n. 10, p. 4010-16, oct. 2000.

RODRIGUES, K. E. S. et al. Atraso diagnóstico do retinoblastoma. Jornal de Pediatria, Rio de Janeiro, v. 80, n. 6, p. 511-16, 2004.

RODRIGUEZ-GAlindO, C. et al. Retinoblastoma: One World, One Vision. Pediatrics,v. 122, n. 3, p. 763-70, 2008.

SHIELDS, C. L. et al. Basic understanding of current classification and management of retinoblastoma. Current Opinion in Ophthalmology, v. 17, p. 228-34, 2006.

SHIELDS, C. L. et al. Retinoblastoma. Revista da Sociedade Brasileira de Retina e Vítreo, v. 8, p. 10-15, 2004.

SHIELDS, J. A. et al. Lesions simulating retinoblastoma. Journal of Pediatric Ophthalmology and Strabismus, v. 28, p. 338-40, 1991.

SZILÁRD, K. et al. Diagnosis, Classification and Treatment of Retinoblastoma. International Ophthalmology Clinics, v. 48, n. 2, p. 135-47, 2008. 